\title{
Mistaken identity? Visual similarities of marine debris to natural prey items of sea turtles
}

\author{
Qamar A Schuyler ${ }^{1 *}$, Chris Wilcox², Kathy Townsend ${ }^{3}$, B Denise Hardesty $^{2}$ and N Justin Marshall ${ }^{4}$
}

\begin{abstract}
Background: There are two predominant hypotheses as to why animals ingest plastic: 1) they are opportunistic feeders, eating plastic when they encounter it, and 2) they eat plastic because it resembles prey items. To assess which hypothesis is most likely, we created a model sea turtle visual system and used it to analyse debris samples from beach surveys and from necropsied turtles. We investigated colour, contrast, and luminance of the debris items as they would appear to the turtle. We also incorporated measures of texture and translucency to determine which of the two hypotheses is more plausible as a driver of selectivity in green sea turtles.

Results: Turtles preferred more flexible and translucent items to what was available in the environment, lending support to the hypothesis that they prefer debris that resembles prey, particularly jellyfish. They also ate fewer blue items, suggesting that such items may be less conspicuous against the background of open water where they forage.

Conclusions: Using visual modelling we determined the characteristics that drive ingestion of marine debris by sea turtles, from the point of view of the turtles themselves. This technique can be utilized to determine debris preferences of other visual predators, and help to more effectively focus management or remediation actions.
\end{abstract}

Keywords: Chelonia mydas, Chromatic space, Eretmochelys imbricata, Marine debris, Vorobyev-Osorio model

\section{Background}

Sea turtles, like many other marine taxa, are increasingly prone to marine debris ingestion and associated problems [1]. Despite many studies recording instances of debris ingestion e.g. [2,3], little is known about the cues that attract turtles to eat plastic debris. The predominant hypotheses are that 1) turtles, as opportunistic feeders, simply consume items in proportion to what they encounter in the environment, including plastics; and 2) that turtles feed on plastic because of its similarity to prey; particularly jellyfish $[4,5]$. Though the proportion of gelatinous prey in a turtle's diet varies depending on the life stage and the species of the turtle, all species do target these prey at some stage of their lives [6,7].

Turtles are primarily visual predators. Research indicates that loggerhead turtles have limited ability to find food based on chemical stimuli alone [8], which may explain why they are primarily caught during the day on longline fishing lines, and rarely at night [9]. Similarly, when presented with both chemical and visual cues, leatherback

\footnotetext{
* Correspondence: Q.Schuyler@uq.edu.au

'School of Biological Sciences, University of Queensland, St. Lucia, Australia Full list of author information is available at the end of the article
}

turtles responded exclusively to visual cues [10]. Therefore, the visual similarity between plastic bags and jellyfish can cause confusion even in the absence of chemical stimuli associated with food sources. Loggerhead sea turtles have been shown to approach plastic bags in a similar manner to gelatinous prey, indicating that they use visual characteristics to select their food [11].

The spectral sensitivity of an animal depends not only on its photopigments, but also on the transmissivity of the ocular media and, in the case of turtles, of the oil droplets associated with the cones. Turtles have a well-developed visual system with at least three different photopigments, indicating the ability to see colour [12]. The visual system of sea turtles is similar to that of fresh water turtles; however, the sea turtles' visual pigments are slightly shifted towards the shorter wavelengths, due to the differences in spectral characteristics of the waters in which the different animals live [13]. Sea turtles generally inhabit clearer, oceanic waters, whereas fresh water contains many dissolved organics and sediments, shifting the maximum light transmission to longer wavelengths [13-15]. The bulk of sea turtle vision studies to date have been conducted on green (Chelonia mydas) and 
loggerhead (Caretta caretta) sea turtles e.g. [16,17]. Liebman and Granda found 3 photopigments in the green turtle retina absorbing maximally at $440 \mathrm{~nm}$ (SWS), $502 \mathrm{~nm}$ (MWS), and $562 \mathrm{~nm}$ (LWS) [18]. Recent evidence indicates that green turtles are also likely to have a fourth, ultraviolet sensitive (UVS) photo-pigment, like their freshwater relatives [17]. Turtles possess at least four different types of oil droplets, again indicating they have four spectral sensitivities, like birds [17]. Each type of oil droplet may be associated with a specific photopigment, or may combine with different photopigments to produce multiple cone receptor types $[14,19]$.

Turtles, like many other vertebrates, also possess double cones, a specialized structure consisting of two cones joined together [20]. The function of the double cone is still unknown; however it has been hypothesized in both birds and reptiles to play a role in discriminating between levels of luminosity or brightness [20-23]. Although the exact composition of the double cone structure is unknown, in fresh water turtles both of the members that make up the double cone have LWS photoreceptors [19].

We created a chromatic space model of the green turtle visual system (sensu [24]) to investigate the following questions: Are green, hawksbill, and flatback turtles selectively ingesting particular types of debris over others, and if so, what characteristics of that debris (colour, texture, translucency, luminance, or background contrast) are most relevant to turtles' foraging choices?

\section{Results}

Our visual model resulted in peak sensitivities of 365, 440, 515, and 560-565 (Figure 1). The mixed effects modelling results indicate that sea turtles select the debris they ingest based on a variety of physical properties. In fact, debris ingested by turtles was significantly different from beach debris for all environmental variables investigated with the exception of background contrast and the contribution of the UV cone (Table 1). Turtles differentiated items most strongly based on their luminance $(\mathrm{p}<0.001$, selectivity ratio $=0.640)$, flexibility $(\mathrm{p}<0.001$, selectivity ratio $=0.437)$, and translucency $(\mathrm{p}=0.001$, selectivity ratio $=0.290$ ). Items ingested by turtles tend to be less bright (i.e. lower luminance value), more flexible, and more translucent than items found in the environment. With respect to wavelengths, items ingested by turtles had significantly lower short wavelength spectrum values $(\mathrm{p}<0.001$, selectivity ratio $=0.215)$.

A simple inspection of the turtle visual space models (Figure 2) shows the difference in the wavelengths of ingested debris and beach debris. The average value of debris ingested by turtles is lower in the short wavelength spectrum than that of beach debris, indicating that the items turtles eat are less blue than what is available to them in the environment.

There were no significant differences observed between plastics ingested by sea turtles of different life history stages (new recruits and juvenile turtles) with respect to the factors tested (colour, texture, translucency, luminance, and background contrast). However, hawksbill and flatback turtles did exhibit some significant differences compared to green turtles. Because we only had a small sample size for hawksbills $(\mathrm{n}=2)$ and flatbacks $(\mathrm{n}=1)$, we omitted them from analyses.

\section{Discussion}

The spectral sensitivities we calculated $(365,440,515$ and 560-565) are well matched with previously published electroretinography (ERG) data of C. mydas spectral

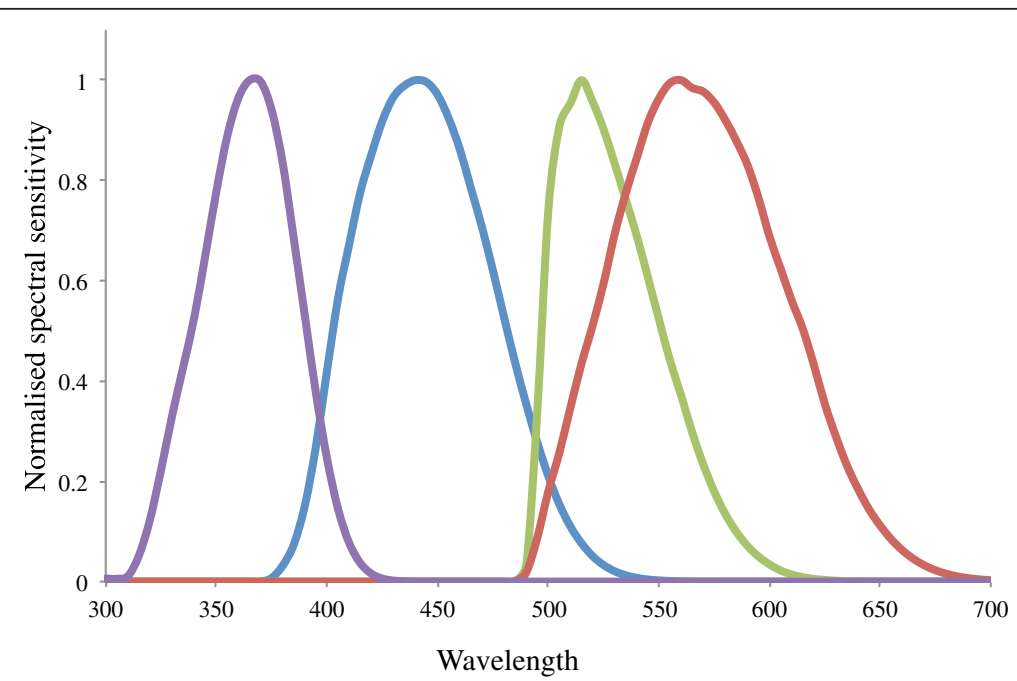

Figure 1 Modelled spectral sensitivity of $\boldsymbol{C}$. mydas. Each peak represents the photopigment multiplied by the transmissivity of its associated oil droplet and by the ocular media. 
Table 1 Model coefficients for physical factors influencing the selectivity of debris ingestion by sea turtles

\begin{tabular}{lllllll}
\hline & Intercept & SE of intercept & Turtle effect & SD of turtle effect & p-value $^{\text {Selectivity ratio }}$ \\
\hline Flexibility & 1.755 & 0.088 & 0.767 & 0.133 & $<0.001^{*}$ & 0.437 \\
Translucency & 1.295 & 0.069 & 0.375 & 0.104 & $0.001^{*}$ & 0.290 \\
SWS & 0.268 & 0.006 & -0.058 & 0.010 & $<0.001^{*}$ & 0.215 \\
MWS & 0.291 & 0.005 & 0.028 & 0.008 & $0.002^{*}$ & 0.096 \\
LWS & 0.311 & 0.008 & 0.040 & 0.012 & $0.002^{*}$ & 0.127 \\
UVS & 0.130 & 0.007 & -0.010 & 0.010 & 0.345 & 0.075 \\
Contrast & 25.981 & 1.551 & -1.468 & 2.356 & 0.573 & 0.057 \\
Luminance (sum of cones) & 239.27 & 16.225 & -153.144 & 24.569 & $<0.001^{*}$ & 0.640 \\
Luminance (double cone) & 74.978 & 4.930 & -43.441 & 7.47 & $<0.001^{*}$ & 0.579 \\
\hline
\end{tabular}

Note that the selectivity ratio indicates the relative strength of the turtles' selectivity based on each factor. *indicate $\mathrm{p}$ values that are significant at the 0.05 level. ${ }^{a}$ Calculated as the absolute value of the ratio of the size of the turtle effect to the size of the intercept.

sensitivities. Levenson and colleagues [25] observed welldefined peaks at 515 and 570, with a relatively constant sensitivity below $500 \mathrm{~nm}$; an earlier study found peaks at 450, 520, and 600 [18]. The technique of high frequency flicker ERG used by Levenson et al. [25] is likely more accurate in the longer wavelengths, as it more successfully isolates the cone response from the rod response. However, the turtles in this study were older than those used by Liebman and Granda and may have experienced a decline in short wavelength vision similar to elderly humans, explaining the lack of a defined short wavelength peak [25]. Our model, therefore, matches observed sensitivities based on ERG.

We assumed that beach debris was a reasonable proxy for ocean-borne debris in the nearshore area inhabited by these turtles, and therefore represents the debris "available" to the turtles. Although there are limitations of using beach debris as a proxy for ocean debris, it has been widely used in previous studies [26]. Thiel et al. [27] conducted a multi-year comparison of anthropogenic marine debris on beaches and in nearshore waters, finding the proportions of different items to be similar. Locally, an analysis of beach debris and nearshore trawl debris for locations around North Stradbroke Island found similar proportions of different colors of debris in both beach and trawl surveys (unpublished observations, Q. Schuyler). We are therefore confident that local beach debris is representative of nearshore ocean debris available to turtles analysed here.

It is clear from the statistical results, as well as from inspection of the turtle visual space data that turtles are selective in what they eat (Table 1, Figure 2). Turtles do not tend to ingest debris that is reflective in the short wavelengths; i.e. blue items. When turtle preferences were analysed based on a human categorical description of colour rather than a turtle visual space model, blue was similarly found to be less prevalent in turtle samples than in beach surveys [28]. Also in support of our findings, a laboratory-based study of loggerhead and Kemp's ridley turtles indicated that both species avoided blue dyed bait [29].
Colour is not the only visual factor employed in food selection. In other animal species, contrast has been found to be as important or even more influential than colour in selecting food sources [30,31]. The fact that turtles are selecting against blue items could indicate that blue plastics are less readily visible against the blue background of the open ocean. We measured this contrast value by calculating the tetrachromatic distance between each debris item and a background measurement of open ocean water, but found that turtles did not selectively ingest items based on contrast. However, this may be partially due to limitations of the model. Similar models calculating colour space distances have reliably predicted honeybee behaviour when visiting orchid mimics. Bees were more likely to visit an orchid mimic when there was a small colour distance between the orchid and its preferred food source than when the colour space distance was large; in other words, when the mimic was a similar colour to their preferred food choice [32]. However, the honeybee model was only successful when incorporating second order visual processing, assuming interactions between photoreceptor types [33]. Our model did not incorporate these interactions, which may explain why turtles did not appear to select for high contrast items.

Turtles selected debris with significantly lower luminance values than those of beach debris, possibly because dark objects stand out better against the bright ocean background [34]. However, we cannot completely exclude the possibility that the prevalence of darker objects in the turtles is partially an artefact of our study design, as the debris in the turtles' gastrointestinal system is exposed to digestive fluids and other waste, which may result in a reduction of luminance. Further work on clarifying the differences in selectivity between contrast and colour would help to elucidate these results.

The visual space model investigates colour and luminance, but other characteristics influence ingestion selectivity in turtles even more than colour. Turtles select plastics most strongly based on their flexibility and 


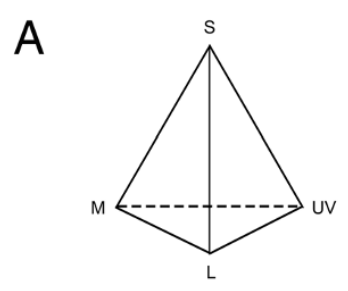

B
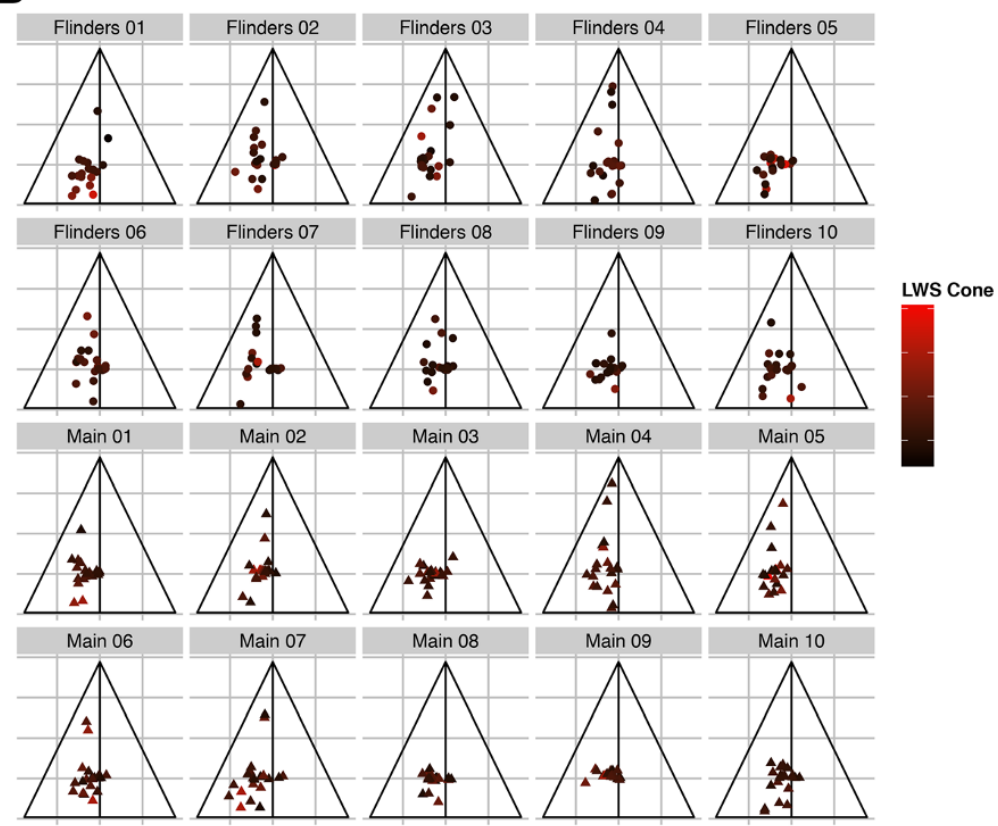

C
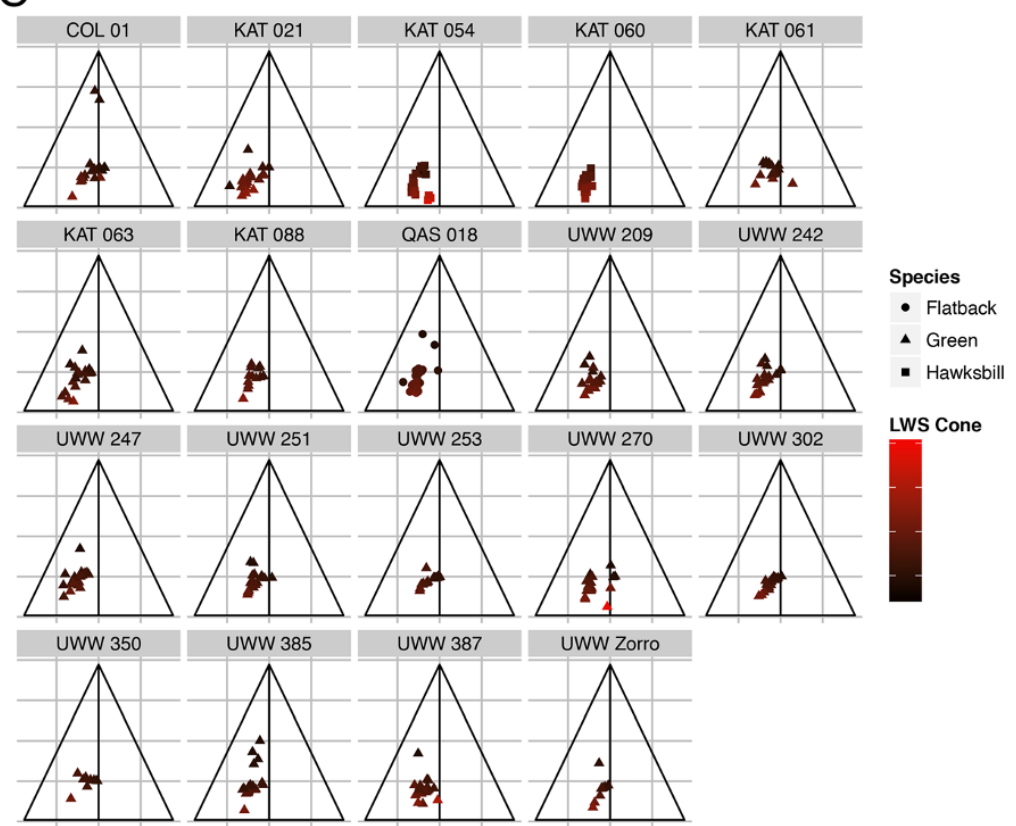

Figure $\mathbf{2}$ (See legend on next page.) 
translucency. Our model suggests that turtles prefer highly flexible and translucent objects, both of which are key characteristics of one of their preferred natural prey items: jellyfish. This work demonstrates that turtles are indeed selective, and it also provides support for the widely postulated "jellyfish hypothesis". Proper waste disposal, particularly for common end user items such as plastic bags and other soft, translucent items which are preferentially ingested by marine turtles, may help to reduce the rapidly increasing debris ingestion rates in threatened sea turtles. We hope this research can inform conservation efforts not only for endangered sea turtles, but we also suggest applying similar analyses for other visual predators to investigate the key factors that drive ingestion rates and anthropogenic debris selectivity.

\section{Conclusions}

Using models to visualize how turtles "see" the plastic they ingest, we find strong support for the hypothesis that they ingest plastic because of its resemblance to a typical prey item, jellyfish. Our model can be extended to other species to better understand why wildlife consume plastic and to effectively focus conservation and remediation efforts.

\section{Methods}

\section{Visual system model}

We modelled the spectral sensitivity of the green sea turtle by incorporating measurements of the photopigments, oil droplets, and ocular media. We generated generic spectral photopigment curves [35-37] based on the peak absorbances for the three known green turtle photopigments: $440 \mathrm{~nm}, 502 \mathrm{~nm}$, and $562 \mathrm{~nm}$ [18]. Since measurements of the green turtle UVS pigment have not been conducted, we simulated a UVS curve based on the UVS pigment of the freshwater turtle Pseudomys scripta. As freshwater turtles tend to have pigment maxima at longer wavelengths than sea turtles, we shifted the peak absorbance for the Pseudomys UVS curve $7 \mathrm{~nm}$ shorter to $365 \mathrm{~nm}$ [19].

For oil droplet measurements, we assumed that the orange oil droplets were associated only with photoreceptors containing the LWS visual pigments, yellow with the MWS, clear (UV-reflective) with the SWS pigments, and colourless (UV-transmissive) with the UVS photoreceptors. We used published curves for yellow and orange oil droplets from green turtles [18], and clear oil droplets from Pseudomys scripta [19]. We shifted the clear oil droplet spectrum shorter by $15 \mathrm{~nm}$, corresponding to the difference in peak wavelength between the SWS pigments of $P$. scripta and of C. mydas [19]. We were unable to find published spectra for the UV-transmissive oil droplet in turtles, but as it has no significant absorbance above $325 \mathrm{~nm}$, it would not affect the shape of the UV photopigment curve.

We applied the Hart correction to each oil droplet [38], converted to transmissivity, and multiplied the photopigment curve by the transmissivity of its associated oil droplet. We then multiplied the four resulting curves by the transmissivity of the ocular media [17] and normalized the result for each cone to an absorbance maximum of 1 to create a modelled spectral sensitivity curve for green sea turtles.

\section{Debris collection and measurement}

We conducted necropsies on sea turtles stranded in southeast Queensland, Australia, between 2006 and 2013, and collected all pieces of debris that had been ingested by the animals (Table 2). For more details see [28]. Of 115 necropsied animals, nineteen had ingested sufficient quantities of debris for our analysis (16 green turtles, 2 hawksbill turtles, and 1 flatback turtle). To estimate the debris to which animals would have been exposed we conducted ten beach surveys on each of two different ocean-facing beaches on North Stradbroke Island (Flinders Beach and Main Beach) between 2011-2013 (for detailed methodology see [28]). All items of anthropogenic debris over $5 \mathrm{~mm}$ in length between the water line and the dominant vegetation line were collected in a $100 \mathrm{~m}$ transect. We selected 20 random debris subsamples from

Table 2 Characteristics of necropsied turtles

\begin{tabular}{lll}
\hline & All turtles necropsied & Turtles with debris \\
\hline Species & & \\
\hline Green & 88 & 16 \\
Hawksbill & 24 & 2 \\
Flatback & 1 & 1 \\
Loggerhead & 2 & 0 \\
\hline Size class & & \\
\hline Pelagic $(C C L<35 \mathrm{~cm})$ & 22 & 12 \\
Benthic $(C C L>35)$ & 93 & 27 \\
\hline
\end{tabular}


each beach and each turtle sample. Three of the turtles had ingested fewer than 20 items of debris, so for these turtles, all pieces were analysed.

We assigned each piece of debris a measurement of flexibility between 1 (impossible to bend without breaking) and 3 (easily malleable). We also assigned a measure of translucency between 1 (completely opaque) and 3 (possible to read text through the item). We chose translucency and flexibility because they are visual characteristics in addition to colour which might be used for prey selection. Using an Ocean Optics JAZ spectrophotometer we measured the reflectance of each item between $300-800 \mathrm{~nm}$ wavelength. In 49 of the plastic samples we did not dark-calibrate the spectra, so some of the reflectances were slightly below zero. To each of the measurements for these samples we added a constant value (equal to the largest negative value for the sample) in order to ensure that the minimum value was non-negative. Because the negative values were quite small with respect to the maximum reflectances, and represent only a linear shift, this correction factor did not affect the outcome of our modelling.

We used our calculated green turtle spectral sensitivities to model how each item of debris would appear in the turtles' visual space [39]. Because there are virtually no studies on the visual systems of hawksbill and flatback turtles (but see [40]), we used the green turtle spectral sensitivity curves (as modelled above) for all species. The visual space for a tetrachromatic animal can be represented as a three dimensional tetrahedron with one vertex for each cone. Plotting the relative excitation of each photoreceptor within this space generates a representation of the colour of an object as it would appear to a turtle's visual system.

Using the Vorobyev-Osorio noise-limited chromatic space model [41] we also calculated the three-dimensional distances between each piece of debris and a measurement of background colour that turtles would be likely to encounter; open ocean water. This gives an indication of the contrast of each item to the background colour. This calculation relies on an estimate of the proportions of cones present in the retina. Although these data are not known for sea turtles, the proportions of oil droplets are [17], so we assumed the proportions of cones in the retina to be equal to the proportions of oil droplets associated with them. Finally, we calculated two different measures of luminance. For the first we added the total reflectance values for all four cones. Since the double cone may be responsible for luminance discrimination, we calculated a second measurement using the total reflectance of the LWS cone only [19].

In order to determine whether turtles exhibited a selectivity for debris based on the physical characteristics measured (colour, texture, translucency, luminance, and background contrast), we used linear mixed effects models
( $\mathrm{R}$ version 3.0.1, package lme4) [42] with the physical factors as response variables, and the location the plastic was found (turtle or beach) as the predictor variable. In order to control for autocorrelation among plastic items within a beach or stomach sample, we incorporated a random effect for each beach or turtle sample. We also investigated the differences between species and life history stages of turtles with respect to each physical characteristic. Because of the complex nature of the data set, we analysed each factor separately. In order to obtain a relative measurement of the strength of each term, we calculated the absolute value of the ratio of the effect size to the intercept term. Note that the larger the ratio, the more highly selective the turtles are for the variable.

\section{Ethical statement}

Because this research was carried out on dead stranded sea turtles, no ethical approval was required.

\section{Competing interests}

The authors declare that they have no competing interests.

\section{Authors' contributions}

QS carried out the field and lab work and drafted the manuscript. CW assisted in statistical analysis. KT and JM conceived of the study. BDH contributed substantial editing of the manuscript. JM participated in the design and coordination of the study. All authors read and approved the final manuscript.

\section{Acknowledgements}

This work was funded by the ARC Linkage grant LP110200216, CSIRO Wealth from Oceans Flagship and Ecosystem Sciences, Goldring Earthwatch Emerging Marine Scientist Fellowship, Goodman Family Foundation, the Australian Postgraduate Award, and the Shell Social Investment Program. We thank Eunji Choi, Bronson Light, Zoe Gilliam, and countless Earthwatch volunteers for their assistance in data collection, the Plumtree Store for support during manuscript writing, the staff of the Moreton Bay Research Station for help throughout this project, and the anonymous reviewers who provided valuable feedback on the manuscript.

\section{Author details}

${ }^{1}$ School of Biological Sciences, University of Queensland, St. Lucia, Australia. ${ }^{2}$ Wealth from Oceans Flagship Marine and Atmospheric Research, Commonwealth Scientific and Industrial Research Organization, Hobart, Australia. ${ }^{3}$ Moreton Bay Research Station, University of Queensland, Dunwich, Australia. ${ }^{4}$ Queensland Brain Institute, University of Queensland, St. Lucia, Australia.

Received: 3 February 2014 Accepted: 24 April 2014

Published: 9 May 2014

\section{References}

1. Schuyler QA, Hardesty BD, Wilcox C, Townsend K: Global analysis of anthropogenic debris ingestion by sea turtles. Conserv Biol 2014, 28(1):128-139.

2. Tomas J, Guitart R, Mateo R, Raga JA: Marine debris ingestion in loggerhead sea turtles, Caretta caretta from the Western Mediterranean. Mar Pollut Bull 2002, 44(3):211-216.

3. Tourinho PS, Do Sul JAl, Fillrnann G: Is marine debris ingestion still a problem for the coastal marine biota of southern Brazil? Mar Pollut Bull 2010, 60(3):396-401.

4. Carr A: Rips, FADS, and little loggerheads. BioScience 1986, 36(2):92-100.

5. Mrosovsky N, Ryan G, James M: Leatherback turtles: the menace of plastic. Mar Pollut Bull 2009, 58(2):287-289.

6. Shaver DJ: Feeding ecology of wild and head-started Kemp's Ridley sea turtles in South Texas waters. J Herpetol 1991, 25(3):327-334. 
7. Bjorndal K: Foraging ecology and nutrition of sea turtles. In The Biology of Sea Turtles. Edited by Lutz PL, Musick JA. Boca Raton, Florida: CRC Press; 1997:199-232.

8. Southwood A, Higgins B, Brill R, Swimmer Y: Chemoreception in loggerhead sea turtles: an assessment of the feasibility of using chemical deterrents to prevent sea turtle interactions with longline fishing gear. In US Department of Commerce, NOAA Technical Memo NOAA-TM-NMFS-PIFSC-10. Honolulu, HI: U. S. Department of Commerce; 2007:1-17.

9. Baez JC, Real R, Caminas JA: Differential distribution within longline transects of loggerhead turtles and swordfish captured by the Spanish Mediterranean surface longline fishery. J Mar Biol Assoc UK 2007, 87(3):801-803.

10. Constantino MA, Salmon M: Role of chemical and visual cues in food recognition by leatherback posthatchlings (Dermochelys coriacea $\mathrm{L}$ ). Zoology 2003, 106:173-181.

11. Narazaki T, Sato K, Abernathy KJ, Marshall GJ, Miyazaki N: Loggerhead turtles (Caretta caretta) use vision to forage on gelatinous prey in mid-water. PLoS One 2013, 8(6).

12. Fritsches KA, Warrant EJ: Vision. In The Biology of Sea Turtles, vol III. vol. 3rd edition. Edited by Wyneken J, Lohmann KJ, Musick JA. New York: CRC Press; 2013.

13. Lythgoe JN: The Ecology of Vision. Oxford: Clarendon Press; 1979.

14. Granda A: Eyes and their sensitivity to light of differing wavelengths. In Turtles: Perspectives and Research. Edited by Warless M, Morlock H. New York: John Wiley and Sons; 1979:247-266.

15. Jerlov NG: Marine Optics. New York: Elsevier; 1976.

16. Bartol SM, Musick JA: Morphology and topographical organization of the retina of juvenile loggerhead sea turtles (Caretta caretta). Copeia 2001 3:718-725.

17. Mäthger $L M$, Litherland $L$, Fritsches KA: An anatomical study of the visual capabilities of the green turtle, Chelonia mydas. Copeia 2007, 1:169-179.

18. Liebman PA, Granda A: Microspectrophotometric measurements of visual pigments in two species of turtle, Pseudemys scripta and Chelonia mydas. Vision Res 1971, 11:105-114.

19. Loew ER, Govardovskii Vl: Photoreceptors and visual pigments in the red eared turtle, Trachemys scripta elegans. Vis Neurosci 2001, 18:753-757.

20. Marchiafava PL: Cell coupling in double cones of the fish retina. Proc R Soc Lond B Biol Sci 1985, 226(1243):211-215.

21. Marshall NJ, Jennings K, McFarland WN, Loew ER, Losey GS: Visual biology of Hawaiian coral reef fishes. III. Environmental light and an integrated approach to the ecology of reef fish vision. Copeia 2003, 3:467-480.

22. Bowmaker J: Visual pigments of fishes. In The Visual System of Fish. Edited by Douglas R, Djamgoz M. Netherlands: Springer; 1990:81-107.

23. Loew ER, Lythgoe JN: The ecology of cone pigments in teleost fishes. Vision Res 1978, 18(6):715-722.

24. Kelber A, Vorobyev M, Osorio D: Animal colour vision — behavioural tests and physiological concepts. Biol Rev 2003, 78(1):81-118.

25. Levenson DH, Eckert SA, Crognale MA, Deegan JF II, Jacobs GH: Photopic spectral sensitivity of green and loggerhead sea turtles. Copeia 2004, 4:908-914.

26. Dixon TR, Dixon TJ: Marine litter surveillance. Mar Pollut Bull 1981, 12(9):289-295.

27. Thiel M, Hinojosa IA, Miranda L, Pantoja JF, Rivadeneira MM, Vasquez N: Anthropogenic marine debris in the coastal environment: A multi-year comparison between coastal waters and local shores. Mar Pollut Bull 2013, 71:307-316.

28. Schuyler Q, Hardesty BD, Wilcox C, Townsend K: To eat or not to eat? Debris selectivity by marine turtles. PLoS One 2012, 7(7). doi:10.1371/journal. pone.0040884

29. Swimmer Y, Arauz R, Higgins B, McNaughton L, McCracken M, Ballestero J, Brill R: Food color and marine turtle feeding behavior: Can blue bait reduce turtle bycatch in commercial fisheries? Mar Ecol-Prog Ser 2005, 295:273-278.

30. Schmidt V, Martin Schaefer $\mathrm{H}$, Winkler $\mathrm{H}$ : Conspicuousness, not colour as foraging cue in plant-animal signalling. Oikos 2004, 106(3):551-557.

31. Ginetz R, Larkin P: Choice of colors of food items by rainbow trout (Salmo gairdneri). J Fish Board Canada 1973, 30(2):229-234.

32. Gumbert A, Kunze J: Colour similarity to rewarding model plants affects pollination in a food deceptive orchid, Orchis boryi. Biol J Linn Soc 2001, 72(3):419-433

33. Brandt $R$, Vorobyev M: Metric analysis of threshold spectral sensitivity in the honeybee. Vision Res 1997, 37(4):425-439.

34. Lythgoe J: Light and vision in the aquatic environment. In Sensory Biology of Aquatic Animals. Edited by Atema J, Fay R, Popper A, Tavolga W. New York: Springer; 1988:57-82.
35. Stavenga D, Smits R, Hoenders B: Simple exponential functions describing the absorbance bands of visual pigment spectra. Vision Res 1993, 33(8):1011-1017.

36. Hart NS: Avian Photoreceptors. PhD Thesis. Bristol, UK: University of Bristol; 1999.

37. Palacios AG, Goldsmith TH, Bernard GD: Sensitivity of cones from a cyprinid fish (Danio aequipinnatus) to ultraviolet and visible light. Vis Neurosci 1996, 13(3):411-421.

38. Hart N, Vorobyev M: Modelling oil droplet absorption spectra and spectral sensitivities of bird cone photoreceptors. J Comp Physiol A Neuroethol Sens Neural Behav Physiol 2005, 191(4):381-392.

39. Marshall NJ, Cheney K: Color vision and color communication in reef fish In Encyclopedia of Fish Physiology: From Gecxxnome to Environment, Volume 1. Edited by Farrell AP. San Diego: Academic Press; 2011:150-158.

40. Northmore DPM, Granda AM: Ocular dimensions and schematic eyes of freshwater and sea turtles. Visual Neurosci 1991, 7(06):627-635.

41. Vorobyev M, Osorio D: Receptor noise as a determinant of colour thresholds. Proc R Soc Lond B Biol Sci 1998, 265(1394):351-358.

42. R Core Team: R: A Language and Environment for Statistical Computing. Vienna, Austria: R foundation for statistical computing; 2013.

doi:10.1186/1472-6785-14-14

Cite this article as: Schuyler et al:: Mistaken identity? Visual similarities of marine debris to natural prey items of sea turtles. BMC Ecology 2014 14:14.

\section{Submit your next manuscript to BioMed Central and take full advantage of:}

- Convenient online submission

- Thorough peer review

- No space constraints or color figure charges

- Immediate publication on acceptance

- Inclusion in PubMed, CAS, Scopus and Google Scholar

- Research which is freely available for redistribution

Submit your manuscript at www.biomedcentral.com/submit
C) Biomed Central 